\title{
The Cytokine Release Inhibitory Drug CRID3 Targets ASC Oligomerisation in the NLRP3 and AIM2 Inflammasomes
}

\author{
Rebecca C. Coll*, Luke A. J. O’Neill
}

School of Biochemistry and Immunology, Trinity College Dublin, Dublin, Ireland

\begin{abstract}
Background: The Inflammasomes are multi-protein complexes that regulate caspase- 1 activation and the production of the pro-inflammatory cytokine IL-1 $\beta$. Previous studies identified a class of diarylsulfonylurea containing compounds called Cytokine Release Inhibitory Drugs (CRIDs) that inhibited the post-translational processing of IL-1 $\beta$. Further work identified Glutathione S-Transferase Omega 1 (GSTO1) as a possible target of these CRIDs. This study aimed to investigate the mechanism of the inhibitory activity of the CRID CP-456,773 (termed CRID3) in light of recent advances in the area of inflammasome activation, and to clarify the potential role of GSTO1 in the regulation of IL-1 $\beta$ production.

Methodology and Results: In murine bone marrow derived macrophages, CRID3 inhibited IL-1 $\beta$ secretion and caspase 1 processing in response to stimulation of NLRP3 and AIM2 but not NLRC4. CRID3 also prevented AIM2 dependent pyroptosis in contrast to the NLRP3 inhibitors glyburide and parthenolide, which do not inhibit AIM2 activation. Confocal microscopy and Western blotting assays indicated that CRID3 inhibited the formation of ASC complexes or 'specks' in response to NLRP3 and AIM2 stimulation. Co-immunoprecipitation assays show that GSTO1 interacted with ASC.

Significance: These results identify CRID3 as a novel inhibitor of the NLRP3 and AIM2 inflammasomes and provide an insight into the mechanism of action of this small molecule. In addition GSTO1 may be a component of the inflammasome that is required for ASC complex formation.
\end{abstract}

Citation: Coll RC, O'Neill LAJ (2011) The Cytokine Release Inhibitory Drug CRID3 Targets ASC Oligomerisation in the NLRP3 and AIM2 Inflammasomes. PLoS ONE 6(12): e29539. doi:10.1371/journal.pone.0029539

Editor: David M. Ojcius, University of California Merced, United States of America

Received November 6, 2011; Accepted November 30, 2011; Published December 22, 2011

Copyright: @ 2011 Coll, O'Neill. This is an open-access article distributed under the terms of the Creative Commons Attribution License, which permits unrestricted use, distribution, and reproduction in any medium, provided the original author and source are credited.

Funding: This work was funded by Science Foundation Ireland (www.sfi.ie). The funder had no role in study design, data collection and analysis, decision to publish, or preparation of the manuscript.

Competing Interests: The authors have declared that no competing interests exist.

* E-mail: collr@tcd.ie

\section{Introduction}

The production of the pro-inflammatory cytokine interleukin (IL)-1 $\beta$ is a highly regulated process. An initial signal through the activation of pattern recognition receptors such as Toll-like receptors (TLRs) induces pro-IL-1 $\beta$ mRNA synthesis. Pro-IL-1 $\beta$ is biologically inactive and requires processing to generate the active 17 kilodalton $(\mathrm{kDa})$ form that is secreted. Pro-IL-1 $\beta$ can be processed by caspase-1 which itself requires processing to form the active enzyme. The activation of caspase- 1 is mediated by high molecular weight protein complexes termed inflammasomes $[1,2]$. In addition to processing IL- $\beta$ and the related IL- 1 family cytokine IL-18, caspase-1 also plays a role in unconventional protein secretion [3] and mediates a form of cell death called pyroptosis [4].

The Nod-like receptor protein NLRP3 forms the prototypical inflammasome by interacting with the adapter molecule apoptosisassociated speck-like protein containing a CARD (ASG) via its Pyrin domain (PYD). The caspase activation and recruitment domain (CARD) of ASG in turn binds the CARD domain of caspase-1 [4,5]. NLRP3 can be activated in response to a highly diverse range of pathogen, environmental and endogenously derived molecules. Danger molecules such as ATP, pore forming toxins such as nigericin [6], particulates such as monosodium urate crystals [7] and fibrils such as islet amyloid polypeptide [8] are all sensed by NLRP3. NLRP3 is not directly activated but appears to sense an intermediate process or cellular perturbation caused by these molecules. These may include potassium efflux, the release of lysosomal proteases and the generation of reactive oxygen species [9]. NLRP3 protein expression levels are also a limiting step in inflammasome activation. NLRP3 thus requires induction or priming by TLR, NLR, IL-1 or TNF $\alpha$ stimulation [10].

Other NLR proteins such as NLRP1 and NLRC4 also form inflammasomes. NLRC4 senses bacterial flagellin and the rod protein from the type III secretion system apparatus of Gramnegative bacteria. It requires another NLR family member either NAIP5 or NAIP2 to detect its ligands [11,12]. Absent in melanoma-2 (AIM2) is a non-NLR protein that is also capable of forming an inflammasome. AIM2 is a member of the PYHIN protein family that contain PYD domains and Hematopoietic expression, IFN-inducible, nuclear localisation (HIN) domains [13]. AIM2 recognises and directly binds cytosolic dsDNA via its HIN domain and recruits ASC to activate caspase-1. AIM2 is a broad sensor of dsDNA as it recognises viral, bacterial, mammalian and synthetic dsDNA $[14,15,16,17]$.

In a screen for inhibitors of IL- $1 \beta$ production a novel class of sulfonylurea containing compounds were identified. These socalled cytokine release inhibitory drugs or CRIDs (CP-424,174 
and CP-412,245) inhibited the post-translational processing and secretion of IL-1 $\beta$ in response to LPS and ATP in human monocytes [18]. Further studies identified glutathione-S-transferase omega 1 (GSTO1) as a possible target for CRIDs [19].

The discovery of CRIDs predates the discovery of the inflammasomes. In this report we sought to characterise the inhibitory activity of the CRID CP-456,773 (termed CRID3) against multiple inflammasomes. We found that CRID3 inhibited both NLRP3 and AIM2 inflammasomes by preventing ASC oligomerisation. In addition GSTO1 was found to associate with ASC suggesting that it might play a role in inflammasome signalling and could indeed be a target of CRID3.

\section{Results}

\section{CRID3 inhibits NLRP3 dependent IL-1 $\beta$ processing}

The effect of CRID3 on the NLRP3 inflammasome was examined in bone marrow derived macrophages (BMDM). As shown in Figure 1A treatment with CRID3 $(5-100 \mu \mathrm{M})$ dose dependently inhibited the amount of IL- $1 \beta$ produced by BMDM stimulated with lipopolysaccharide (LPS) and the NLRP3 activator ATP, with $50 \mu \mathrm{M}$ having an optimum effect. The inhibition of IL$1 \beta$ secretion was specific as CRID3 did not inhibit TNF $\alpha$ secretion from these cells (Figure 1B). In Figure 1C it was confirmed by Western blotting that GRID3 prevented the ATP stimulated processing of pro-IL-1 $\beta$ to the mature 17 kilodalton $(\mathrm{kDa})$ form, the effect being optimal at $50 \mu \mathrm{M}$ (compare lanes 5-7 to lane 3). As shown in Figure 1D pre-treatment with CRID3 did not affect the induction of pro-IL-1 $\beta$ by LPS (compare lanes 4 and 5 to lane 3).
The AIM2 inflammasome is inhibited by CRID3

As CRID3 was found to inhibit NLRP3 dependent responses we next tested its effect on the activation of other inflammasomes. Figure 2A shows that CRID3 inhibits the release of IL-1 $\beta$ from BMDM stimulated with LPS and the AIM2 activator synthetic dsDNA analog Poly (dA:dT) in a concentration dependent manner, optimum inhibition occurring at $50 \mu \mathrm{M}$. IL-1 $\beta$ secretion was not affected by treatment with the NLRP3 inhibitor glyburide [20] or parthenolide, which has also been shown to inhibit NLRP3 [21]. Glyburide and parthenolide both inhibited NLRP3 activation by LPS and ATP (data not shown). As shown in Figure 2B the secretion of $\mathrm{TNF} \alpha$ from these cells was not significantly inhibited by CRID3 or glyburide, with parthenolide having a partial inhibitory effect. In Figure $2 \mathrm{C}$ treatment with CRID3 prevented the production of mature cleaved IL- $1 \beta$ and also prevented the processing of pro-caspase- 1 as determined by detection of the p10 fragment in the supernatants. This effect was specific to the processing of IL- $1 \beta$ and caspase- 1 as the levels of pro-IL-1 $\beta$ and pro-caspase-1 in the cell lysates were not decreased by treatment with CRID3 (lanes 5 and 6 in each case compared to lane 3).

In addition to cytokine processing AIM2 activation also leads to a caspase-1 dependent form of cell death known as pyroptosis. Figure 2D demonstrates that CRID3 prevents Poly (dA:dT) induced cell death, the effect being evident from $30 \mu \mathrm{M}$. Neither glyburide nor parthenolide inhibited this response. Figure $2 \mathrm{E}$ demonstrates that Poly (dA:dT) stimulated caspase-1 activation is also prevented in the presence of CRID3 (lane 4) but not glyburide (lane 5) or parthenolide (lane 6).
A
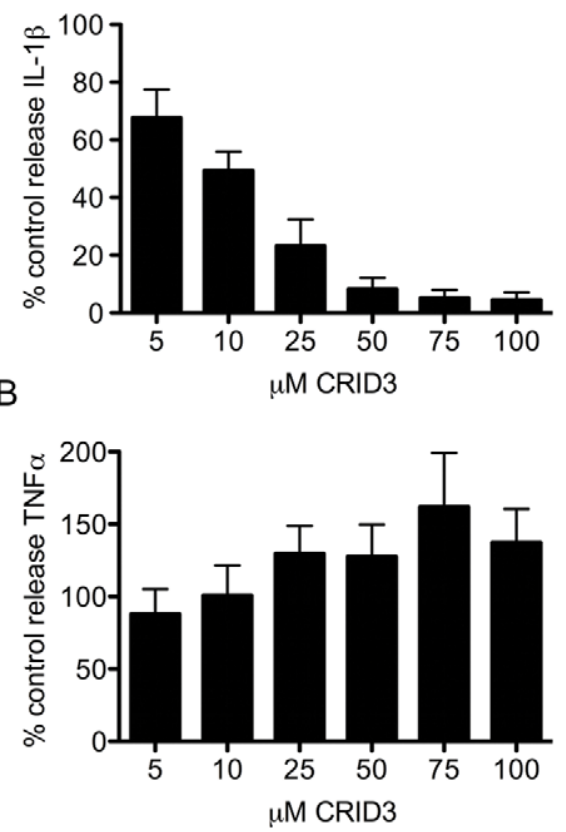

C

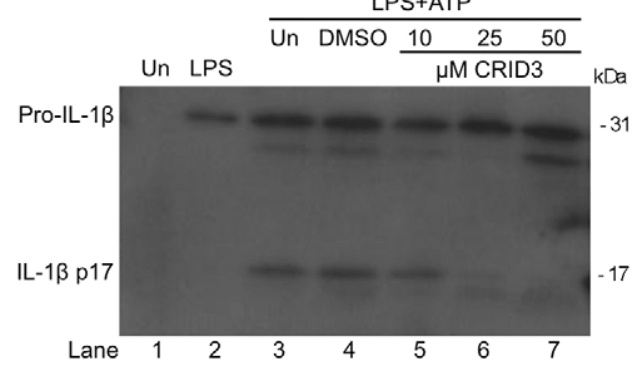

D

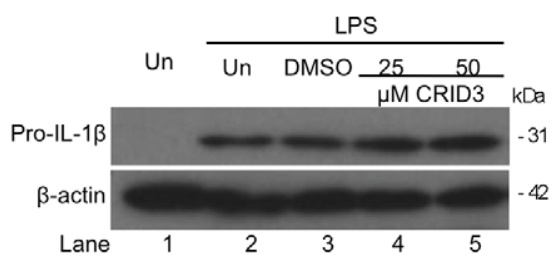

Figure 1. CRID3 inhibits LPS and ATP induced IL-1 $\beta$ processing. Primary BMDM were stimulated with $10 \mathrm{ng} / \mathrm{ml}$ LPS for $3 \mathrm{~h}$, treated with CRID3 $(5-100 \mu \mathrm{M})$ or DMSO in serum free media for $30 \mathrm{~min}$ followed by addition of $5 \mathrm{mM}$ ATP for $1 \mathrm{~h}$. Supernatants were analysed by ELISA for IL $-1 \beta$ (A), TNF $\alpha$ (B) or by Western blotting for pro and mature IL-1 $\beta$ (C). (A) and (B) Cytokine level is expressed as a percentage of that released from control treated cells, which ranged across all experiments from $521-1988 \mathrm{pg} / \mathrm{ml}$ for IL-1 $\beta$ and $104-211 \mathrm{pg} / \mathrm{ml}$ for TNF $\alpha$. Data are expressed as mean \pm S.E.M of five independent experiments each carried out in triplicate. (C) A representative blot from one of three independent experiments is shown. (D) BMDM were treated with DMSO or CRID3 $(25-50 \mu \mathrm{M})$ for $30 \mathrm{~min}$ and were then stimulated with $10 \mathrm{ng} / \mathrm{ml} \mathrm{LPS} \mathrm{for} 3 \mathrm{~h}$. Protein samples were analysed by Western blotting using anti-IL-1 $\beta$ and anti- $\beta$-actin antibodies. A representative blot from one of two independent experiments is shown. doi:10.1371/journal.pone.0029539.g001 
A

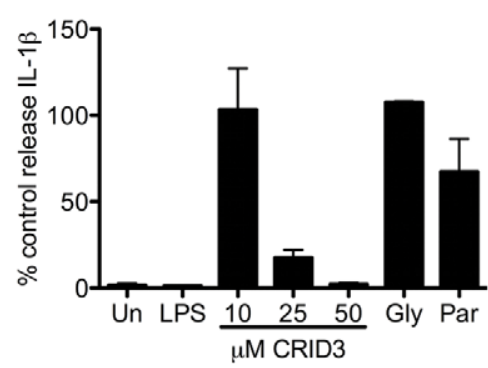

B

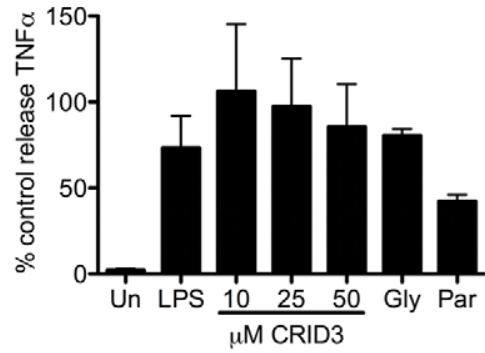

D

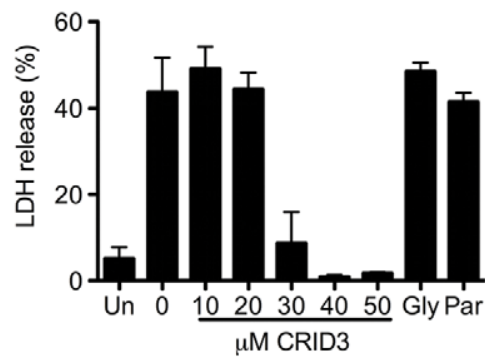

C

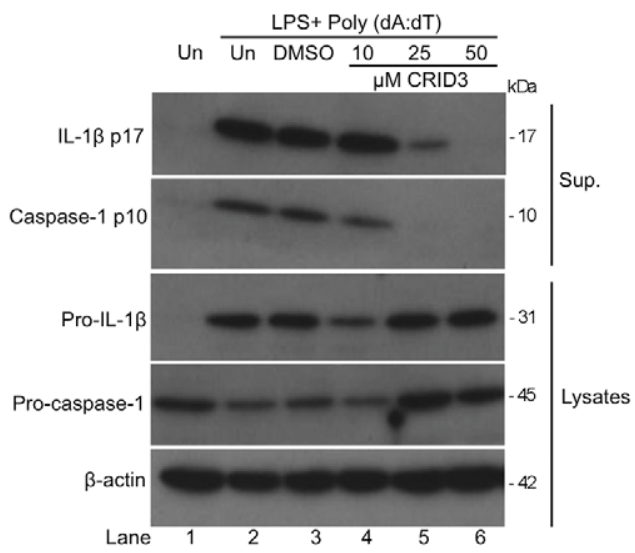

$\mathrm{E}$

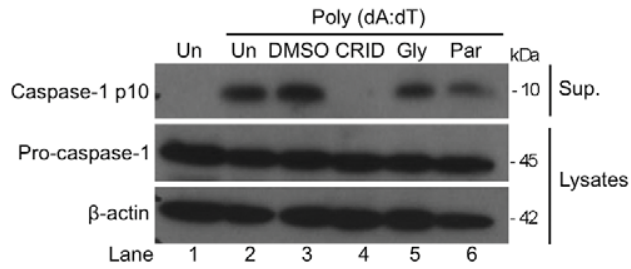

Figure 2. CRID3 inhibits the AIM2 inflammasome. Primary BMDM were stimulated with $10 \mathrm{ng} / \mathrm{ml}$ LPS for $3 \mathrm{~h}$, treated with CRID3 (10-50 $\mu$ M), $200 \mu \mathrm{M}$ glyburide, $10 \mu \mathrm{M}$ parthenolide or DMSO control in serum free media for $1 \mathrm{~h}$ followed by transfection of $1 \mu \mathrm{g} / \mathrm{ml}$ Poly (dA:dT) for $4 \mathrm{~h}(\mathrm{~A})$ and (B) or overnight (C). Supernatants were analysed by ELISA for IL $-1 \beta$ (A) and TNF $\alpha$ (B). (A) and (B) Cytokine level is expressed as a percentage of that released from control treated cells, which ranged across all experiments from 518-2347 pg/ml for IL-1 $\beta$ and $397-1136 \mathrm{pg} / \mathrm{ml}$ for TNF $\alpha$. Data are expressed as mean \pm S.E.M of four independent experiments each carried out in triplicate. In (C) concentrated supernatants and cell lysates were analysed by Western blotting using anti-IL-1 $\beta$, anti-caspase- 1 and anti- $\beta$-actin antibodies. These results are representative of three independent experiments. (D) and (E) Primary BMDM were treated with CRID3 (10-50 $\mu \mathrm{M}), 200 \mu \mathrm{M}$ glyburide, $10 \mu \mathrm{M}$ parthenolide or DMSO in serum free media for $1 \mathrm{~h}$ followed transfection of $1 \mu \mathrm{g} / \mathrm{ml}$ Poly (dA:dT) for $6 \mathrm{~h}$. Supernatants were analysed using an LDH cytotoxicity assay. The data shown represent mean \% LDH release \pm S.D. from triplicate determinations and are representative of three independent experiments. In (E) Concentrated supernatants and cell lysates were analysed by Western blotting using anti-caspase- 1 and anti- $\beta$-actin antibodies. These results are representative of four independent experiments.

doi:10.1371/journal.pone.0029539.g002

\section{CRID3 does not inhibit the NLRC4 inflammasome}

The effect of CRID3 on the activation of the NLRC4 inflammasome was also tested. A previous report showed that in response to infection with Salmonella typhimurium at early time points IL-1 $\beta$ secretion and cell death are NLRC4 dependent [22]. In Figure 3 BMDM were primed with LPS prior to infection with $S$. typhimurium. Pre-treatment with CRID3 or glyburide did not significantly affect the levels of IL-1 $\beta$ (Figure 3A), TNF $\alpha$ (Figure 3B) or LDH (Figure 3C) released by cells. In agreement with the absence of an effect on IL- $1 \beta$ secretion or LDH release, pre-treatment with CRID3 or glyburide did not affect caspase-1 activation (Figure 3D). The levels of IL-1 $\beta$ p17 and caspase-1 p10 detected in the supernatants of cells stimulated with LPS and $S$. typhimurium was similar under every condition tested.

\section{CRID3 prevents NLRP3 and AIM2 dependent ASC oligomerisation}

The activation of NLRP3 and AIM2 results in the recruitment of ASC and the formation of large molecular weight oligomers that in turn activate caspase-1. Figure $4 \mathrm{~A}$ demonstrates that stimulation of immortalized BMDM (I-BMDM) with LPS and ATP caused the formation of ASC dimers and higher order oligomers, which are detected in the cell pellet by Western blotting (bottom panel, compare lanes 2 and 3). However, the pretreatment of cells with CRID3 and parthenolide before stimulation with ATP prevented ASC oligomerisation (bottom panel, lanes 5 and 6 ), the production of IL- $1 \beta$ (top panel lanes 5 and 6) caspase- 1 activation (third panel, lanes 5 and 6). CRID3 and parthenolide did not affect ASC expression, as seen in the cell lysates (fourth panel). As shown in Figure 4B transfection of I-BMDM with Poly (dA:dT) also resulted in the appearance of ASC dimers in cell pellets (bottom panel, lane 2). CRID3 inhibited the activation of caspase-1 (top panel, lane 4) and the formation of ASC oligomers (bottom panel, lane 4) whereas parthenolide and glyburide had no effect on either response (top and bottom panels, lanes 5 and 6).

An additional assay was also employed to examine the activation of ASC oligomerisation by NLRP3 and AIM2. Murine immortalized macrophages that stably express ASC-YFP were used to analyse the formation of ASC oligomers or 'specks'. As shown in Figure 5A in unstimulated cells or cells treated with LPS alone (Figure 5B) the YFP-ASC is diffuse throughout the cytosol of 
A

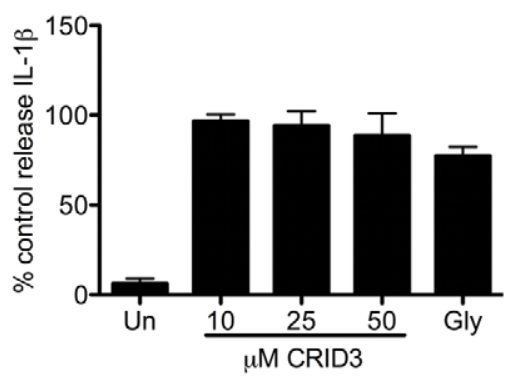

C

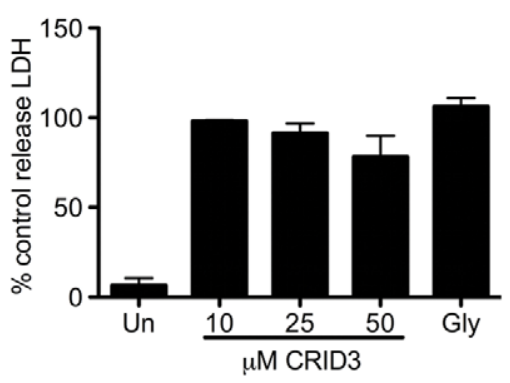

B

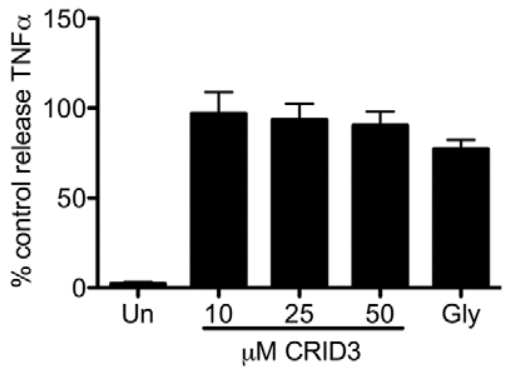

D

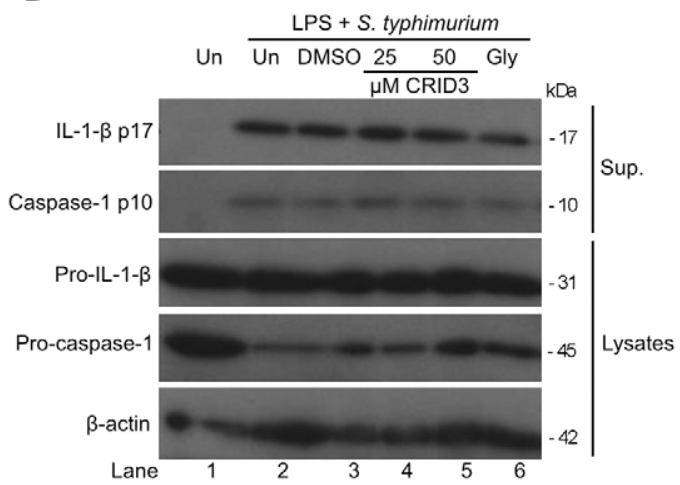

Figure 3. CRID3 and glyburide do not inhibit the NLRC4 inflammasome. Primary BMDM were stimulated with $10 \mathrm{ng} / \mathrm{ml}$ LPS for $3 \mathrm{~h}$, treated with CRID3 $(10-50 \mu \mathrm{M}), 200 \mu \mathrm{M}$ glyburide or DMSO in serum free media for 30 min followed by infection with S. typhimurium (M.O.I 20) for $2 \mathrm{~h}$. Supernatants were analysed by ELISA for IL $-1 \beta(A)$ and TNF $\alpha$ (B) production or by LDH cytotoxicity assay (C). (A) and (B) cytokine level is expressed as a percentage of that released from control treated cells, which ranged across all experiments from 534-3013 pg/ml for IL-1 $\beta$ and $1990-2746 \mathrm{pg} / \mathrm{ml}$ for TNF $\alpha$. (A), (B) and (C) data are expressed as mean \pm S.E.M of three independent experiments each carried out in triplicate. In (D) concentrated supernatants and cell lysates were analysed by Western blotting using anti-IL-1 $\beta$, anti-caspase- 1 and anti- $\beta$-actin antibodies. These results are representative of three independent experiments.

doi:10.1371/journal.pone.0029539.g003

the cell, but in response to stimulation with LPS and ATP (Figure 5C, first row) or Poly (dA:dT) (Figure 5D, first row) the YFP-ASC condenses into a large, bright, speck. Arrowheads are included to indicate typical specks. CRID3 at $50 \mu \mathrm{M}$ and $10 \mu \mathrm{M}$ parthenolide prevented the formation of specks in response to stimulation with LPS and ATP (Figure 5C, second and third rows). As shown in Figure 5D CRID3 $(50 \mu \mathrm{M})$ also inhibited speck formation in cells stimulated with LPS and Poly (dA:dT) (Figure 5dD second row), whereas glyburide had no effect (Figure 5D, third row).

\section{GSTO1 interacts with ASC}

Previous investigations into the target of the CRID compounds had identified GSTO1 as the primary candidate for their target of action [19]. As CRID3 inhibited both NLRP3 and AIM2 dependent responses it was investigated whether GSTO1 could interact with components of the inflammasome. As shown in Figure 6A ASC immunoprecipitated together with both endogenous lane 7) and overexpressed GSTO1 (lane 8) in HEK293T cells. Figure $6 \mathrm{~B}$ shows that no interaction between NLRP3 and GSTOl could be detected (lanes 7 and 8).

\section{Discussion}

This study has identified CRID3 as an inhibitor of the NLRP3 and AIM2 inflammasomes but not the NLRC4 inflammasome. CRID3 inhibited ASC oligomerisation. A previously identified target of CRID3, GSTO1 was shown to interact with ASC suggesting that it may be required for ASC function in the inflammasome.

CRID3 dose dependently prevented IL-1 $\beta$ but not TNF $\alpha$ secretion from BMDM stimulated with LPS and ATP. This result is in line with previous studies on the effects of related CRID compounds $[18,19]$. The decrease in IL- $1 \beta$ secretion was due to inhibition of IL-1 $\beta$ processing as CRID3 did not have an effect on pro-IL-1 $\beta$ induction. CRID3 was also found to inhibit the AIM2 inflammasome, preventing IL-1 $\beta$ secretion, caspase- 1 activation and cell death in response to AIM2 activation by Poly (dA:dT).

The other NLRP3 inhibitors glyburide and parthenolide had no significant effect on AIM2 mediated responses pointing to specificity in their actions. Juliana et al. [21] have shown that parthenolide directly inhibits caspase-1 by alkylation of certain cysteine residues. Our results disagree with this assertion as we did not find that parthenolide inhibited caspase-1 in response to AIM2 stimulation. However, the previous study [21] did not test AIM2 activation so perhaps parthenolide only inhibits caspase- 1 in response to NLRP3 or NLRC4 activation. Importantly CRID3 did not affect NLRC4 mediated caspase-1 activation and IL- $1 \beta$ secretion in response to $S$. typhimurium infection.

It was possible that CRID3 was targeting ASC, since ASG is required for the function of the NLRP3 and AIM2 inflammasomes but has a lesser role in the NLRC4 inflammasome. In both the Western blotting and ASC-YFP fluorescence microscopy assays it was found that pre-treatment with CRID3 prevented ASC oligomerisation in response to stimulation with LPS and ATP or Poly (dA:dT). In agreement with the effects seen previously on IL- 


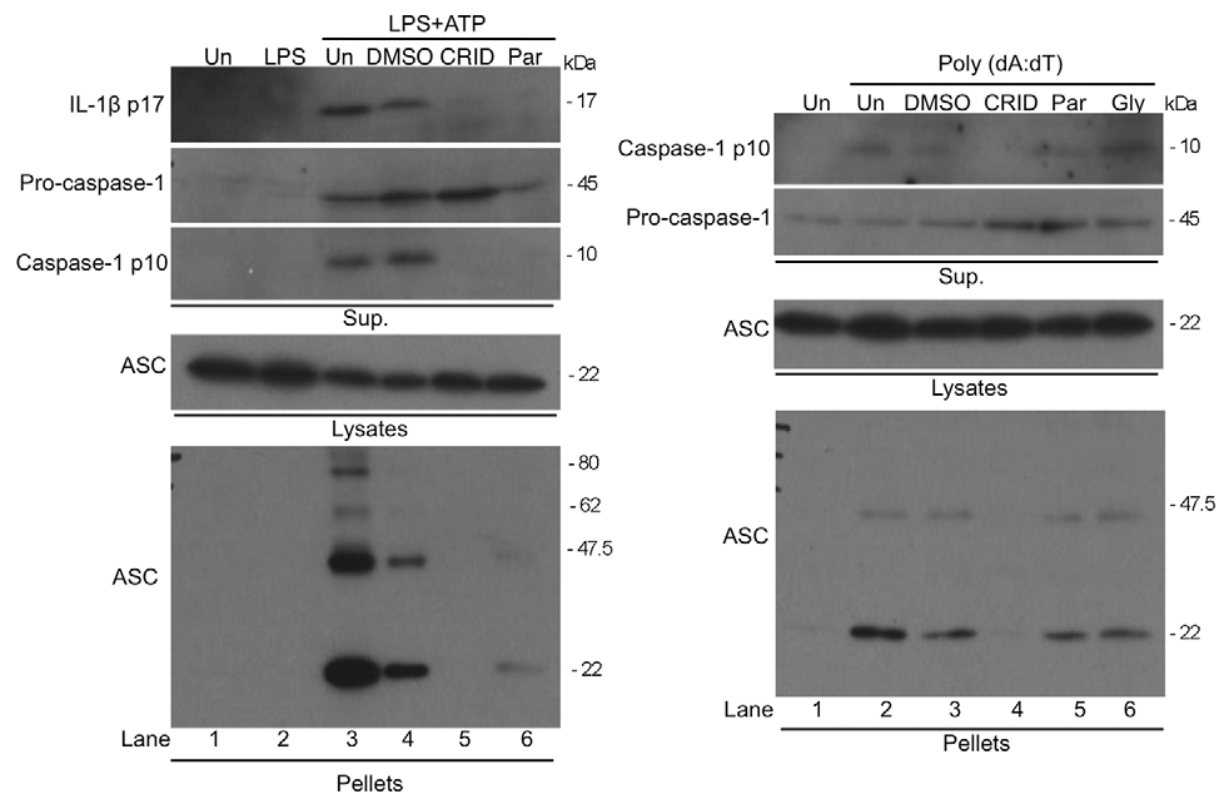

Figure 4. CRID3 prevents NLRP3 and AIM2 stimulated ASC oligomerisation. (A) I-BMDM were stimulated with $10 \mathrm{ng} / \mathrm{ml}$ LPS for $3 \mathrm{~h}$, were treated with $50 \mu \mathrm{M}$ CRID3, $10 \mu \mathrm{M}$ parthenolide, DMSO or were left untreated in serum free media for 30 min followed by addition of 5 mM ATP for $1 \mathrm{~h}$. (B) Cells were treated with with $50 \mu \mathrm{M}$ CRID3, $200 \mu \mathrm{M}$ glyburide, $10 \mu \mathrm{M}$ parthenolide, DMSO or were left untreated in serum free media for $30 \mathrm{~min}$ followed by transfection with $1 \mu \mathrm{g} / \mathrm{ml}$ Poly (dA:dT) for $5 \mathrm{~h}$. Concentrated supernatants and cell lysates were analysed by Western blotting using anti-IL-1 $\beta$ and/or anti-caspase- 1 antibodies. The cell lysates were centrifuged at $330 \mathrm{x} \mathrm{g}$ for $10 \mathrm{~min}$ at $4^{\circ} \mathrm{C}$. The pellets were washed and resuspended in PBS and then cross-linked by incubation with DSS for $30 \mathrm{~min}$. The cross-linked pellets and cell lysates were analysed by Western blotting with anti-ASC and anti-caspase- 1 antibodies. The data shown are representative of two (A) and three (B) independent experiments. doi:10.1371/journal.pone.0029539.g004

$1 \beta$ secretion and caspase- 1 activation glyburide and parthenolide did not inhibit AIM2 dependent ASC oligomerisation.

ASC is indispensible for both NLRP3 and AIM2 inflammasome formation. As CRID3 prevents ASC oligomerisation in response to activation of both these molecules this would suggest that CRID3 acts directly or at the level of ASC in these complexes. The role of ASC in the NLRC4 inflammasome is less clear. The caspase activation and recruitment domain (CARD) of NLRC4 can interact directly with caspase-1 but ASC facilitates more efficient IL-1 $\beta$ secretion $[1,23]$. ASG does not play a role in NLRC4 induced cell death as in vivo studies showed NLRC4 mediated pyroptosis, which is critical for $S$. typhimurium clearance was independent of ASC [24]. CRID3 did not prevent $S$. typhimurium induced cell death and thus does not inhibit NLRC4. There may be a partial role for ASC in IL- $1 \beta$ induction in response to NLRC4, since at early time points this response is impaired in ASC deficient macrophages [22]. However we found that CP- CRID3 had no effect on NLRC4 dependent IL-1 $\beta$ secretion. This may be because ASC was not involved in the particular conditions we tested or because CRID3 specifically inhibits ASC on the NLRP3 and AIM2 pathways. From our ASC assays however, it is clear that CRID3 can inhibit ASC activation by NLRP3 and AIM2.

A previous study using affinity labelling and affinity binding chromatography techniques had identified GSTO1 as the target of CRID compounds [19]. GSTO are the most recently identified and characterised class of cytosolic GSTs. GSTO1 is an unusual GST as it contains an active site cysteine residue and displays little activity with typical GST substrates. It does however have thiol transferase activity that is characteristic of glutaredoxins [25]. An initial possible mechanism of CRID3 inhibition could have been through directly preventing caspase-1 activation. A previous study has shown that caspase-1 could be inhibited by glutathionylation of Cys397 and Cys362 during conditions of oxidative stress [26]. Thus it was possible that CRID3 could deglutathionylate caspase1. However, as CRID3 failed to inhibit NLRC4 activated caspase1 activation this suggested that CRID3 was not directly affecting caspase-1. It has been suggested previously that related CRID compounds were not direct caspase- 1 inhibitors although the data was not shown in the report [18]. The physiological role of GSTO1 remains ill-defined although some studies have implicated a role in drug resistance and the oxidative stress response $[27,28]$. Polymorphisms in GSTO1 were also shown to affect the risk of developing sporadic Alzheimer's disease [29].

We speculated that GSTO1 might interact with components of the inflammasome. Co-immunoprecipitation experiments indicated that GSTO1 did not interact with NLRP3 but did interact with ASC. GSTO1 is therefore a novel component of the NLRP3 and AIM2 inflammasomes. How GSTO1 affects ASC is open to speculation. As mentioned previously caspase-1 was shown to be negatively regulated by glutathionylation [26], ASC may also subject to this post-translational modification. There is an emerging literature indicating that reversible glutathionylation of proteins is a form of signal transduction analogous to protein phosphorylation or ubiquitination.

Glutathionylation has been implicated in the regulation of $\mathrm{TNF} \alpha$ induced apoptosis through reversible glutathionylation of caspase-3 [30]. While the glutathionylation of endothelial nitric oxide synthase (eNOS) has been shown to regulate its function in vasodilation [31]. There are also examples of GSTs as regulators of cytokine signalling. GSTP regulates TNF $\alpha$ signalling by associating with tumour-necrosis factor receptor-associated factor 
A

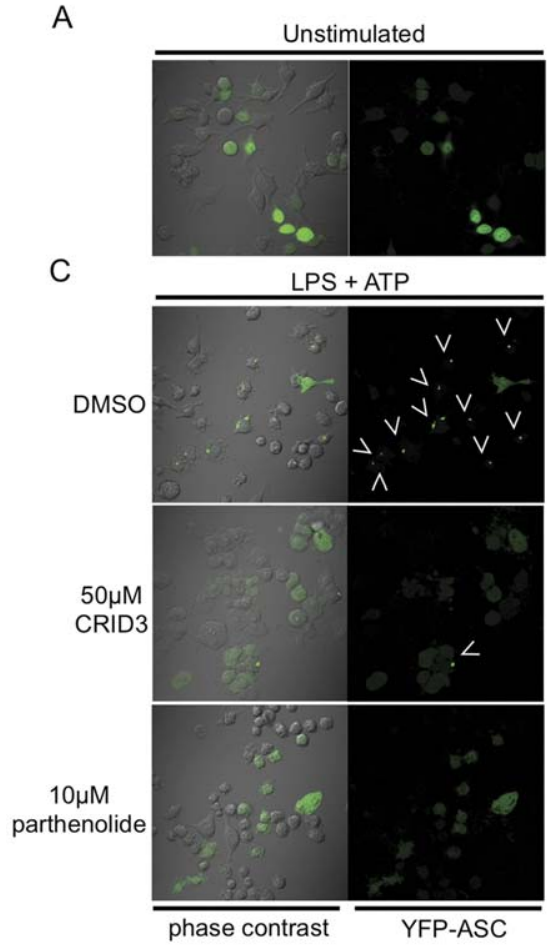

B
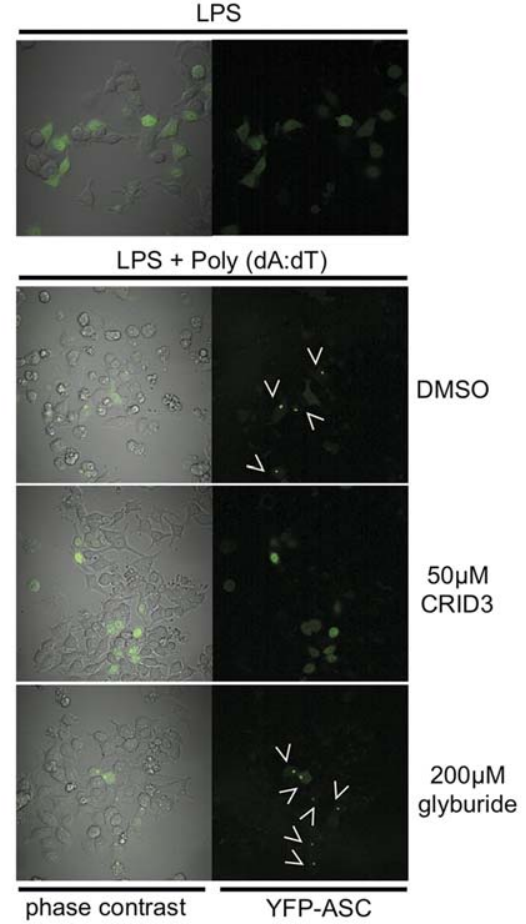

Figure 5. CRID3 inhibits NLRP3 and AIM2 stimulated ASC speck formation. (A) ASC-YFP cells were left unstimulated, or were (B) stimulated with $10 \mathrm{ng} / \mathrm{ml} \mathrm{LPS} \mathrm{for} 3 \mathrm{~h}$. (C) Cells were stimulated with $10 \mathrm{ng} / \mathrm{ml}$ LPS for $3 \mathrm{~h}$, treated with $50 \mu \mathrm{M}$ CRID3 $10 \mu \mathrm{M}$ parthenolide or DMSO in serum free media for $30 \mathrm{~min}$ followed by addition of $5 \mathrm{mM}$ ATP for $1 \mathrm{~h}$. In (D) cells were stimulated with $10 \mathrm{ng} / \mathrm{ml}$ LPS for $3 \mathrm{~h}$, treated with $50 \mu \mathrm{M}$ CRID3, $200 \mu \mathrm{M}$ glyburide or DMSO in serum free media for 40 min followed by transfection of $1 \mu \mathrm{g} / \mathrm{ml}$ Poly (dA:dT) for $2 \mathrm{~h}$. Cells were viewed by live cell imaging, at least four different images were taken of each dish of which a representative image is shown. These results are representative of three independent experiments.

doi:10.1371/journal.pone.0029539.g005

2 (TRAF2) and blocking the TRAF2 interaction with apoptosis signal regulating kinase 1 (ASK1). This activity of GSTP was independent of its glutathione conjugating activity [32]. Perhaps GSTO1 regulates the interaction of ASC with NLRP3 or AIM2 or with other ASC molecules, with CRID3 interfering with this interaction, preventing formation of the inflammasome protein complex.

There is significant evidence that ASC regulates innate immune responses separate to its role in the inflammasome. Studies on ASC deficient mice have shown that ASC is required for the induction of humoral immunity after vaccination with the MF59 adjuvant. ASC deficient mice had significantly reduced antigen specific IgG responses, whereas caspase-1 and NLRP3 deficient mice had normal responses [33]. In contrast to caspase-1 and NLRP3 deficient mice, ASC deficient mice were completely protected from the development of arthritis in a collagen induced arthritis study. This was due to defective $\mathrm{T}$ cell activation by ASC deficient dendritic cells [34]. ASG deficient mice were also more protected than caspase- 1 deficient mice from a mouse model of multiple sclerosis (experimental autoimmune encephalitis or EAE) [35]. An inhibitor of ASC such as CRID3 could therefore be an effective treatment for these diseases. A recent study has also defined an inflammasome independent role for ASC in the posttranscriptional regulation of the guanine nucleotide exchange factor Dock2. ASC deficient dendritic cells had very low Dock2 expression, which led to decreased Rac dependent actin polymerization and consequently impaired antigen uptake and chemotaxis [36]. As GSTO1 interacted with ASC but not NLRP3 it is possible that GSTOl may also influence these non- inflammasome related functions of ASC. Further studies on the mechanism of ASC function in these settings may establish if GSTO1 is required specifically or ubiquitously for ASC mediated immune responses.

Given the role of IL-1 $\beta$ in diseases such as type 2 diabetes [8], gout [7] and osteoarthritis [37], which all involve NLRP3, a small molecule such as CRID3 could have promise therapeutically and be an alternative to IL-1 $\beta$ biologics such as the anti-IL-1 $\beta$ antibody Canakinumab and the IL-1 Trap Rilonacept. Future work aims to elucidate the precise molecular target for CRID3.

\section{Materials and Methods}

\section{Materials}

Ultrapure rough LPS (from E. coli, serotype EH100) was purchased from Alexis. Poly(deoxyadenylic-thymidylic) acid sodium salt (Poly dA:dT) and adenosine 5'-triphosphate disodium salt hydrate (ATP) were purchased from Sigma-Aldrich. $S$. typhimurium UK-1 strain was obtained from Dr. Sinead Corr (Trinity College Dublin, Ireland). Glyburide was obtained from Sigma-Aldrich. Parthenolide was purchased from Enzo Life Sciences. CP-456,773 (CRID3) was from Amgen Inc., Thousand Oaks, CA, USA. GeneJuice ${ }^{\circledR}$ was purchased from Novagen. Lipofectamine $2000^{\mathrm{TM}}$ was from Invitrogen. Protein A/G Plus agarose immunoprecipitation reagent was from Santa Cruz Biotechnology. StrataClean ${ }^{\mathbf{T M}}$ resin was from Agilent Technologies. TNF- $\alpha$ and IL-1 $\beta$ ELISA Duosets were purchased from R\&D Systems. CytoTox $96^{\circledR}$ non-radioactive cytotoxicity assay was from Promega. 
A

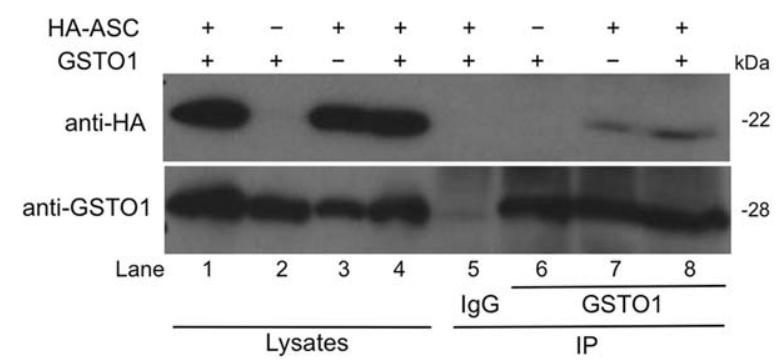

B

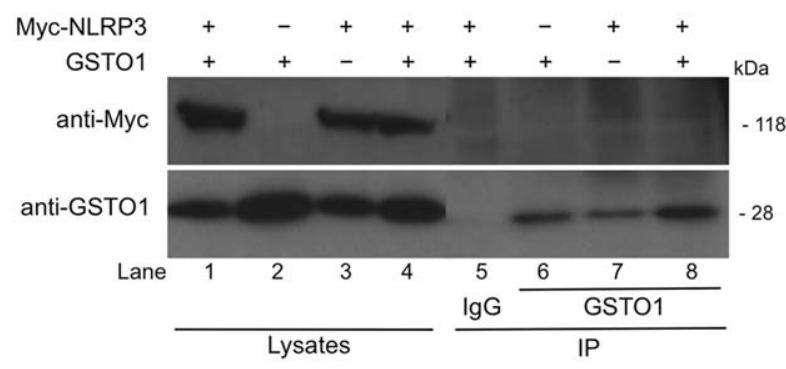

Figure 6. ASC but not NLRP3 immunoprecipitates with GSTO1. HEK-293T cells were transfected with HA-ASC, Myc-NLRP3, GSTO1 or empty vector plasmids as indicated. 48 hours post transfection cells were lysed in low stringency lysis buffer. $50 \mu \mathrm{l}$ of lysate was kept for analysis while the remaining lysates were pre-cleared using $10 \mu \mathrm{l}$ Protein $A / G$ beads for $30 \mathrm{~min}$. The lysates were then incubated with anti-GSTO1 antibody or rabbit lgG coupled to protein A/G beads for $3 \mathrm{~h}$ at $4{ }^{\circ} \mathrm{C}$. Whole cell lysates and immunoprecipitation samples were analysed by Western blotting using anti-Myc, anti-HA or anti-GSTO1 antibodies. The results presented are representative of two (B) and three $(A)$ independent experiments.

doi:10.1371/journal.pone.0029539.g006

Human GSTO1 pcDNA3.1 was a gift from Prof. Philip Board, John Curtin School of medical research, Australian National University, Australia. The pCI ASC-HA expression vector was a gift from Dr. Kate Fitzgerald, University of Massachusetts, USA. Empty vector pcDNA 3.1 myc-His was from Invitrogen. Myccaspase-1 and myc-NLRP3 were gifts from Prof. Dan Kastner, National Institute of Arthritis and Musculoskeletal and Skin Diseases, Bethesda, Maryland, USA. ASC antibody (AL177) was from Enzo Life Sciences. Murine caspase-1 pl0 (M-20) and c-Myc (9E10) antibodies were obtained from Santa Cruz Biotechnology. HA.11 antibody was from Covance. Murine IL-1 $\beta$ antibody was from R\&D Systems. Rabbit antiserum raised against recombinant hGSTO1-1 was a gift from Prof. Philip Board, John Curtin School of medical research, Australian National University, Australia. $\beta$ actin, and anti-rabbit IgG were obtained from Sigma-Aldrich.

\section{Cell culture}

The immortalised BMDM were a kind gift from Prof. Douglas Golenbock, University of Massachusetts, USA [38]. Stably transfected ASC-FP macrophages were a kind gift from Dr. Eicke Latz, University of Massachusetts, USA [39]. The human embryonic kidney T cell line (HEK-293T) was purchased from the European Cell Culture Collection. HEK293T, ASC-FP and IBMDM cell lines were cultured in Dulbecco's modified Eagle's medium (DMEM) supplemented with 10\% fetal calf serum (FCS) and $1 \%$ penicillin/streptomycin (v/v). Bone marrow from C57BL/6 mice was differentiated for 7 days in DMEM supplemented with $10 \%$ fetal calf serum (FCS), 1\% penicillin/ streptomycin (v/v) and M-CSF (20\% (v/v) L929 mouse fibroblast supernatant). For experiments the differentiated BMDM were seeded at $0.25-1 \times 10^{6} / \mathrm{ml}$.

\section{Enzyme-linked immunosorbent assay}

For cytokine measurements, BMDM were stimulated in triplicate as indicated. Supernatants were removed and analysed for IL- $1 \beta$ and TNF- $\alpha$ using enzyme-linked immunosorbent assay (ELISA) kits according to the manufacturer's instructions (R\&D Systems).

\section{Western blotting}

For Western blotting experiments BMDM were seeded in 12 or 6 well plates at $0.5-1 \times 10^{6} / \mathrm{ml}$. Cell lysates were prepared by direct lysis in 5X Laemmli sample buffer. The protein content of supernatants was concentrated using StrataClean ${ }^{\mathrm{TM}}$ resin according to the manufacturer's instructions. The protein samples were resolved on $12 \%$ or $15 \%$ SDS-PAGE gels and transferred onto polyvinylidene diflouride (PVDF) membrane using a wet transfer system. Membranes were blocked in 5\% (w/ v) dried milk in TBS-T (50 mM Tris/HCL, pH 7.6, $150 \mathrm{mM}$ $\mathrm{NaCl}$ and $0.1 \%(\mathrm{v} / \mathrm{v})$ Tween-20) for an hour at room temperature (RT). The membranes were incubated with primary antibody diluted 1 in 1000 in $5 \%(w / v)$ dried milk in TBS-T. The membranes were then incubated with the appropriate horse radish peroxidise conjugated secondary antibody diluted 1 in 2000 in 5\% (w/v) dried milk in TBS-T for an hour before being developed by enhanced chemiluminescence (ECL) according to the manufacturer's instructions (Cell Signaling Technology, Inc.). Some membranes were stripped using Restore ${ }^{\mathrm{TM}}$ PLUS western blot stripping buffer according to the manufacturer's instructions (Thermo Fisher Scientific Inc.) before being reprobed.

\section{Cytotoxicity assay}

A CytoTox $96{ }^{\circledR}$ non-radioactive cytotoxicity assay kit was used according to the manufacturer's instructions (Promega) to determine lactate dehydrogenase $(\mathrm{LDH})$ release from cells.

\section{ASC complex isolation}

$2 \times 10^{6} \mathrm{I}$-BMDM cells were seeded in each well of a 6 well plate. The following day cells were stimulated with $10 \mathrm{ng} / \mathrm{ml}$ LPS for 3 hours before treatment with the indicated inhibitors or controls for 30 mins followed by the addition of $5 \mathrm{mM}$ ATP for 1 hour or cells were pre-treated with inhibitors for $30 \mathrm{mins}$ followed by transfection of $1 \mu \mathrm{g} / \mathrm{ml}$ Poly (dA:dT) for $5 \mathrm{hrs}$. The supernatants were removed, cells were rinsed in ice-cold PBS and $500 \mu \mathrm{l}$ icecold buffer (20 mM Hepes-KOH, pH 7.5, $150 \mathrm{mM} \mathrm{KCL,} \mathrm{1 \%}$ NP-40 $0.1 \mathrm{mM}$ PMSF, $1 \mu \mathrm{g} / \mathrm{ml}$ leupeptin, $11.5 \mu \mathrm{g} / \mathrm{ml}$ aprotinin and $1 \mathrm{mM}$ sodium orthovanadate) was added. The cells were removed using a cell scraper and transferred to microcentrifuge tubes. Cells were lysed by shearing $10 \mathrm{X}$ through a 21 gauge needle. $50 \mu$ of lysate was removed for Western blot analysis. The lysates were centrifuged at $330 \mathrm{xg}$ for $10 \mathrm{~min}$ at $4^{\circ} \mathrm{C}$. The pellets were washed twice in $1 \mathrm{ml}$ ice-cold PBS (centrifuged $330 \mathrm{x} \mathrm{g}$ for $3 \mathrm{~min}$ at $4^{\circ} \mathrm{C}$ ) and resuspended in $500 \mu \mathrm{l} \mathrm{PBS}$. $2 \mathrm{~mm}$ disuccinimydyl suberate (DSS) (from a fresh $100 \mathrm{mM}$ stock prepared from DSS equilibrated to RT and made up in dry DMSO) was added to the re-suspended pellets, which were incubated at RT for $30 \mathrm{~min}$ with rotation. The samples were then centrifuged at $330 \mathrm{x} g$ for $10 \mathrm{~min}$ at $4^{\circ} \mathrm{C}$. The supernatant was removed and the cross-linked pellets were resuspended in $30 \mu \mathrm{l} \mathrm{Laemmli} \mathrm{sample} \mathrm{buffer.} \mathrm{The}$ samples were boiled for $5 \mathrm{~min}$ at $99^{\circ} \mathrm{C}$ and analysed by Western blotting. 


\section{Confocal microscopy}

ASC-FP cells were seeded at $2 \times 10^{5} / \mathrm{ml}$ the day prior to use in experiments on $35 \mathrm{~mm}$ glass bottom culture dishes. Cells were stimulated with $10 \mathrm{ng} / \mathrm{ml}$ LPS for 3 hours before treatment with the indicated inhibitors or controls for 30-40 mins followed by the addition of $5 \mathrm{mM}$ ATP for 1 hour or transfection of $1 \mu \mathrm{g} / \mathrm{ml}$ Poly (dA:dT) for $2 \mathrm{hrs}$. Imaging was performed on an Olympus FluoView ${ }^{\text {TM }}$ FV1000 Microscope equipped with a temperature and $\mathrm{CO}_{2}$ controlled chamber.

\section{Co-immunoprecipitation assay}

HEK293T cells were seeded at $2 \times 10^{5}$ per ml. 24 hours later cells were transfected with a total of $8 \mu \mathrm{g}$ of the indicated plasmids using GeneJuice ${ }^{\circledR}$. Cells were cultured for 36-48 hours prior to harvesting in low stringency lysis buffer $(50 \mathrm{mM}$ Hepes $\mathrm{pH} 7.5$, $100 \mathrm{mM} \mathrm{NaCl}, 1 \mathrm{mM}$ ethylenediaminetetraacetic acid (EDTA), $10 \%$ glycerol, $0.5 \%$ Nonidet P40 (NP-40), $1 \mathrm{mM}$ phenylmethylsulphonyl fluoride (PMSF), $1 \mu \mathrm{g} / \mathrm{ml}$ leupeptin, $11.5 \mu \mathrm{g} / \mathrm{ml}$ aprotinin and $1 \mathrm{mM}$ sodium orthovanadate] on ice for $15 \mathrm{~min}$. Lysates were centrifuged at $1610 \mathrm{xg}$ for $10 \mathrm{~min}$ at $4^{\circ} \mathrm{C}$. Lysates were pre-cleared for 30 min with $10 \mu \mathrm{l}$ Protein A/G Plus agarose beads. Prior to incubation with lysates $1 \mu \mathrm{g}$ of the relevant antibodies was incubated with $25 \mu$ l Protein A/G Plus agarose beads overnight. The lysates were then incubated with precoupled antibodies/beads for $4 \mathrm{hrs}$ rotating at $4^{\circ} \mathrm{C}$. The lysates and beads were centrifuged at $80 \times \mathrm{g}$ for $3 \mathrm{~min}$ at $4^{\circ} \mathrm{C}$, the supernatant was removed and the beads were washed 3 times in $1 \mathrm{ml}$ of low stringency lysis buffer. The immune complexes were eluted by the addition of $35 \mu \mathrm{l}$ Laemmli sample buffer, and analysed by SDS-PAGE and Western blotting.

\section{Acknowledgments}

We would like to thank Amgen for the provision of CRID3 and Professor Phillip Board for the kind gift of GSTO1 reagents.

\section{Author Contributions}

Conceived and designed the experiments: RCG LAJO. Performed the experiments: RCG. Analyzed the data: RCG LAJO. Wrote the paper: RCG LAJO.

\section{References}

1. Bauernfeind F, Ablasser A, Bartok E, Kim S, Schmid-Burgk J, et al. (2011) Inflammasomes: current understanding and open questions. Cellular and molecular life sciences: CMLS 68: 765-783.

2. Schroder K, Tschopp J (2010) The inflammasomes. Cell 140: 821-832.

3. Keller M, Ruegg A, Werner S, Beer HD (2008) Active caspase-1 is a regulator of unconventional protein secretion. Cell 132: 818-831.

4. Fernandes-Alnemri T, Wu J, Yu JW, Datta P, Miller B, et al. (2007) The pyroptosome: a supramolecular assembly of ASC dimers mediating inflammatory cell death via caspase-1 activation. Cell death and differentiation 14: 1590-1604.

5. Agostini L, Martinon F, Burns K, McDermott MF, Hawkins PN, et al. (2004) NALP3 forms an IL-1beta-processing inflammasome with increased activity in Muckle-Wells autoinflammatory disorder. Immunity 20: 319-325.

6. Mariathasan S, Weiss DS, Newton K, McBride J, O'Rourke K, et al. (2006) Cryopyrin activates the inflammasome in response to toxins and ATP. Nature 440: 228-232.

7. Martinon F, Petrilli V, Mayor A, Tardivel A, Tschopp J (2006) Gout-associated uric acid crystals activate the NALP3 inflammasome. Nature 440: 237-241.

8. Masters SL, Dunne A, Subramanian SL, Hull RL, Tannahill GM, et al. (2010) Activation of the NLRP3 inflammasome by islet amyloid polypeptide provides a mechanism for enhanced IL-1beta in type 2 diabetes. Nature immunology 11: 897-904.

9. Latz E (2010) The inflammasomes: mechanisms of activation and function. Current opinion in immunology 22: 28-33.

10. Bauernfeind FG, Horvath G, Stutz A, Alnemri ES, MacDonald K, et al. (2009) Cutting edge: NF-kappaB activating pattern recognition and cytokine receptors license NLRP3 inflammasome activation by regulating NLRP3 expression. J Immunol 183: 787-791.

11. Kofoed EM, Vance RE (2011) Innate immune recognition of bacterial ligands by NAIPs determines inflammasome specificity. Nature.

12. Zhao Y, Yang J, Shi J, Gong YN, Lu Q et al. (2011) The NLRC4 inflammasome receptors for bacterial flagellin and type III secretion apparatus. Nature.

13. Unterholzner L, Keating SE, Baran M, Horan KA, Jensen SB, et al. (2010) IFI16 is an innate immune sensor for intracellular DNA. Nature immunology 11: $997-1004$.

14. Roberts TL, Idris A, Dunn JA, Kelly GM, Burnton CM, et al. (2009) HIN-200 proteins regulate caspase activation in response to foreign cytoplasmic DNA. Science 323: 1057-1060.

15. Fernandes-Alnemri T, Yu JW, Datta P, Wu J, Alnemri ES (2009) AIM2 activates the inflammasome and cell death in response to cytoplasmic DNA. Nature 458: 509-513.

16. Hornung V, Ablasser A, Charrel-Dennis M, Bauernfeind F, Horvath G, et al. (2009) AIM2 recognizes cytosolic dsDNA and forms a caspase-1-activating inflammasome with ASC. Nature 458: 514-518.

17. Burckstummer T, Baumann C, Bluml S, Dixit E, Durnberger G, et al. (2009) An orthogonal proteomic-genomic screen identifies AIM2 as a cytoplasmic DNA sensor for the inflammasome. Nat Immunol 10: 266-272.

18. Perregaux DG, McNiff P, Laliberte R, Hawryluk N, Peurano H, et al. (2001) Identification and characterization of a novel class of interleukin-1 posttranslational processing inhibitors. The Journal of pharmacology and experimental therapeutics 299: 187-197.

19. Laliberte RE, Perregaux DG, Hoth LR, Rosner PJ, Jordan CK, et al. (2003) Glutathione s-transferase omega 1-1 is a target of cytokine release inhibitory drugs and may be responsible for their effect on interleukin-1beta posttranslational processing. The Journal of biological chemistry 278: 16567-16578.

20. Lamkanfi M, Mueller JL, Vitari AC, Misaghi S, Fedorova A, et al. (2009) Glyburide inhibits the Cryopyrin/Nalp3 inflammasome. The Journal of cell biology 187: 61-70.

21. Juliana C, Fernandes-Alnemri T, Wu J, Datta P, Solorzano L, et al. (2010) Antiinflammatory compounds parthenolide and Bay 11-7082 are direct inhibitors of the inflammasome. The Journal of biological chemistry 285: 9792-9802.

22. Broz P, von Moltke J, Jones JW, Vance RE, Monack DM (2010) Differential requirement for Caspase-1 autoproteolysis in pathogen-induced cell death and cytokine processing. Cell host \& microbe 8: 471-483.

23. Poyet JL, Srinivasula SM, Tnani M, Razmara M, Fernandes-Alnemri T, et al. (2001) Identification of Ipaf, a human caspase-1-activating protein related to Apaf-1. The Journal of biological chemistry 276: 28309-28313.

24. Miao EA, Leaf IA, Treuting PM, Mao DP, Dors M, et al. (2010) Caspase-1induced pyroptosis is an innate immune effector mechanism against intracellular bacteria. Nature immunology 11: 1136-1142.

25. Board PG, Coggan M, Chelvanayagam G, Easteal S, Jermiin LS, et al. (2000) Identification, characterization, and crystal structure of the Omega class glutathione transferases. The Journal of biological chemistry 275: 24798-24806.

26. Meissner F, Molawi K, Zychlinsky A (2008) Superoxide dismutase 1 regulates caspase-1 and endotoxic shock. Nature immunology 9: 866-872.

27. Piaggi S, Raggi G, Corti A, Pitzalis E, Mascherpa MC, et al. (2010) Glutathione transferase omega 1-1 (GSTO1-1) plays an anti-apoptotic role in cell resistance to cisplatin toxicity. Carcinogenesis 31: 804-811.

28. Burmeister C, Luersen K, Heinick A, Hussein A, Domagalski M, et al. (2008) Oxidative stress in Caenorhabditis elegans: protective effects of the Omega class glutathione transferase (GSTO-1). The FASEB journal: official publication of the Federation of American Societies for Experimental Biology 22: 343-354.

29. Capurso C, Panza F, Seripa D, Frisardi V, Imbimbo BP, et al. (2010) Polymorphisms in glutathione S-transferase omega-1 gene and increased risk of sporadic Alzheimer disease. Rejuvenation research 13: 645-652.

30. Pan S, Berk BC (2007) Glutathiolation regulates tumor necrosis factor-alphainduced caspase- 3 cleavage and apoptosis: key role for glutaredoxin in the death pathway. Circulation research 100: 213-219.

31. Chen CA, Wang TY, Varadharaj S, Reyes LA, Hemann C, et al. (2010) Sglutathionylation uncouples eNOS and regulates its cellular and vascular function. Nature 468: 1115-1118.

32. Wu Y, Fan Y, Xue B, Luo L, Shen J, et al. (2006) Human glutathione Stransferase P1-1 interacts with TRAF2 and regulates TRAF2-ASK1 signals. Oncogene 25: 5787-5800.

33. Ellebedy AH, Lupfer C, Ghoneim HE, DeBeauchamp J, Kanneganti TD, et al. (2011) Inflammasome-independent role of the apoptosis-associated speck-like protein containing CARD (ASC) in the adjuvant effect of MF59. Proceedings of the National Academy of Sciences of the United States of America 108: 2927-2932.

34. Ippagunta SK, Brand DD, Luo J, Boyd KL, Calabrese C, et al. (2010) Inflammasome-independent role of apoptosis-associated speck-like protein containing a CARD (ASG) in T cell priming is critical for collagen-induced arthritis. The Journal of biological chemistry 285: 12454-12462.

35. Shaw PJ, Lukens JR, Burns S, Chi H, McGargill MA, et al. (2010) Cutting edge: critical role for PYCARD/ASG in the development of experimental autoimmune encephalomyelitis. Journal of immunology 184: 4610-4614. 
36. Ippagunta SK, Malireddi RK, Shaw PJ, Neale GA, Walle LV, et al. (2011) The inflammasome adaptor ASC regulates the function of adaptive immune cells by controlling Dock2-mediated Rac activation and actin polymerization. Nature immunology 12: 1010-1016.

37. Jin C, Frayssinet P, Pelker R, Cwirka D, Hu B, et al. (2011) NLRP3 inflammasome plays a critical role in the pathogenesis of hydroxyapatiteassociated arthropathy. Proceedings of the National Academy of Sciences of the United States of America 108: 14867-14872.
38. Kenny EF, Talbot S, Gong M, Golenbock DT, Bryant CE, et al. (2009) MyD88 adaptor-like is not essential for TLR2 signaling and inhibits signaling by TLR3. J Immunol 183: 3642-3651.

39. Hornung V, Bauernfeind F, Halle A, Samstad EO, Kono H, et al. (2008) Silica crystals and aluminum salts activate the NALP3 inflammasome through phagosomal destabilization. Nat Immunol 9: 847-856. 